\title{
PSAV MODEL: AGILE METHOD TO DESIGN HUMAN MACHINE INTERACTION FOR USER EXPERIENCE ACROSS
}

THE PLATFORMS

\author{
ABHIJEET SHUKLA \\ Master of Design, User Experience Designer and Analyst, \\ Indian Institute of Technology Kanpur, Tata Consultancy Services, India
}

Translation of design insights structured as a feature and functionalities in the user centered design process, it needs iterations to arrange and decide visual elements. This paper talks about the novel model of organizing insights and design elements to bridge the gap between functional design requirements as well as technical implementation in any digital application development.

KEYWORDS: Agile Design Methodology, User Centered Design, Human Machine Interaction \& User Experience
\end{abstract}

Received: Oct 05, 2018; Accepted: Oct 25, 2018; Published: Nov 15, 2018; Paper Id.: IJCNWMCDEC20182

\section{INTRODUCTION TO PSAV METHOD}

The introduced method focuses on bridging the user's mental model to that of designer's mental model for any scenario in Human Machine Interaction design. It is a process of design which helps designers personalize the user required functions in order to-

- To arrange personalized tasks and functions for users.

- To align the design elements as the primary sensory input for the users.

- To align these objectives to the selection criteria, which provides the switch for action to the user.

- To propagate the flow through action elements in order to proceed towards the process completion.

- To align the perceived information with the final output of the process.

\section{PSAV MODEL (PROCESS)}

- The PSAV model process helps designers to understand the requirements of the design and extract them out into the matrix of "PSAV".

- PSAV design method intent to reduce the cognitive load from the user and directs them towards making a decision or the action required to proceed which is followed by validation of the action results.

- IOT, Service design, enterprise application, interfaces for medical devices, in brief the objective oriented Human Machine Interaction applications are not meant to engage the user for a long time, especially for the purpose of finding and figuring out what to do and when to do and how to do it. 


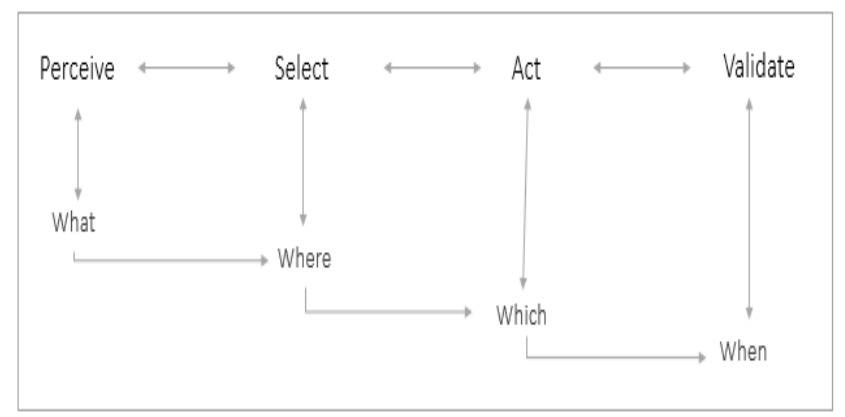

Figure 1: PSAV Process and Key Reasoning Points

\section{PHILOSOPHY OF THE METHOD}

For completion of any process the cognitive challenges of what, why, where $\&$ when put unnecessary cognitive load on the users which hinder the decision of selection. It is a set of a process model and a matrix which provides a guided path towards the visibility of the content for the user to operate/interact with.

There is no one way to practice design methods. John Chris Jones once recognized this by stating:

"Methodology should not be a fixed track to a fixed destination, but a conversation about everything that could be made to happen. The language of the conversation must bridge the logical gap between past and future, but in doing so it should not limit the variety of possible futures that are discussed nor should it force the choice of a future that is unfree."

PSAV method focuses on minimizing the diversions for the user by providing the simple interactions and interface points.The philosophical purpose of this method is to develop a strategy in design operations in the competitive existence of the products, for managing design related requirements, too fast process the design meta steps.

\section{PSAV MODEL MATRIX}

In this section paper talks about the Model matrix, an illustration of the matrix is shown in Figure 2 below.

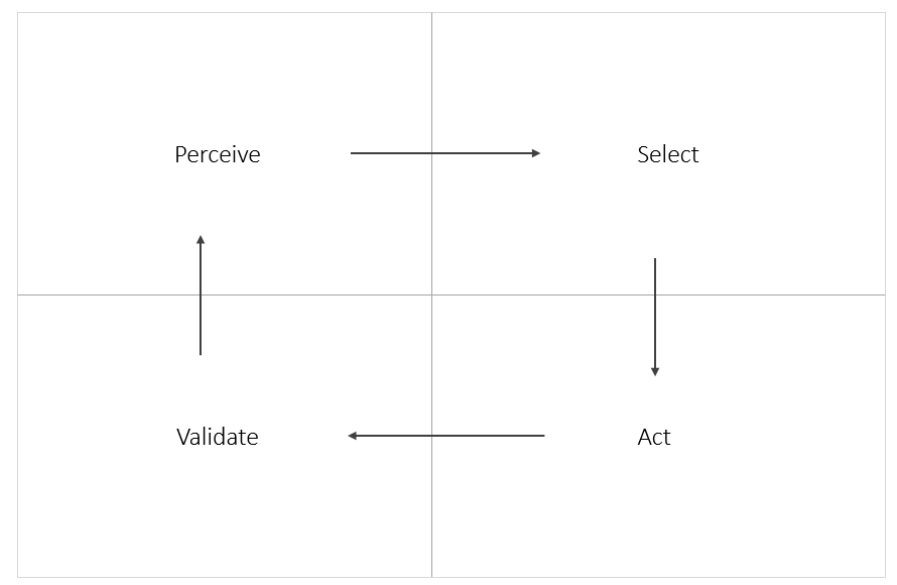

Figure 2: PSAV Data Arrangement Model Matrix

- Quadrant of 'Perceive': In this quadrant designers are supposed to write all the elements which convey-

- Objective of the interactive process

- Required interaction for the user 
- Functionalities that lead towards the next step of the process.

- Interactive, functional, objective, specific information in any media format suitable for the user.

The purpose of this Perceive quadrant is to classify and chart out all the requirements/needs which may lead towards the process initiation. The elements in the perceive quadrant have the basic characteristics of defining "What" the user should perceive

- Quadrant of 'Select': A design can have as many perspectives as many user there could be, but for certain there are at least two perspectives, one of the designer's and one of the user's. Shortest route of jumping to the next step of the process must be available for the user. As shown in fig- 1PSAV method has applicability in defining any application of multiple layers. These layers can comprise of three basic kinds-

- Flow system

- Meta process of User flow

- Interaction definition

The purpose of this quadrant is to organize the keys/Switches which are meant to initiate an active process. It can be identified as the trigger for the action initiation. These trigger receive the command from the user to proceed to the next step. These triggers are inventive in nature and must be able, to interact with the user as well as capture the exact interactive commands in response.

- Quadrant of 'Act': It is a progressive section of 'select', where a selection has been made by a user and now system is required to produce/ present the correct actionable interaction or information to proceed. The actions can be as follows-

- Arrangement of actions requirements

- Type of actions

- Action sequence

- Quadrant of Validation: Once an action has been performed system should be able to validate the action results. This validation must also be extended to the user in the preferred perceivable format. In this section the validation key, commands and messages in the format preferable to users are required to be present.

\section{COMPATIBILITY ESTABLISHMENT}

PSAV model is an agile design methodology which enables a designer to create a highly interactive system design at the macro and micro level as shown in Figure 3. PSAV process has its applicability in the area of user experience design and concerned areas of Human Computer Interaction design. Key compatibilities of the method are listed below-

- PSAV method is compatible with the Lean Framework of development.

- PSAV method is compatible with Agile Framework

- PSAV method is compatible User centric design process 


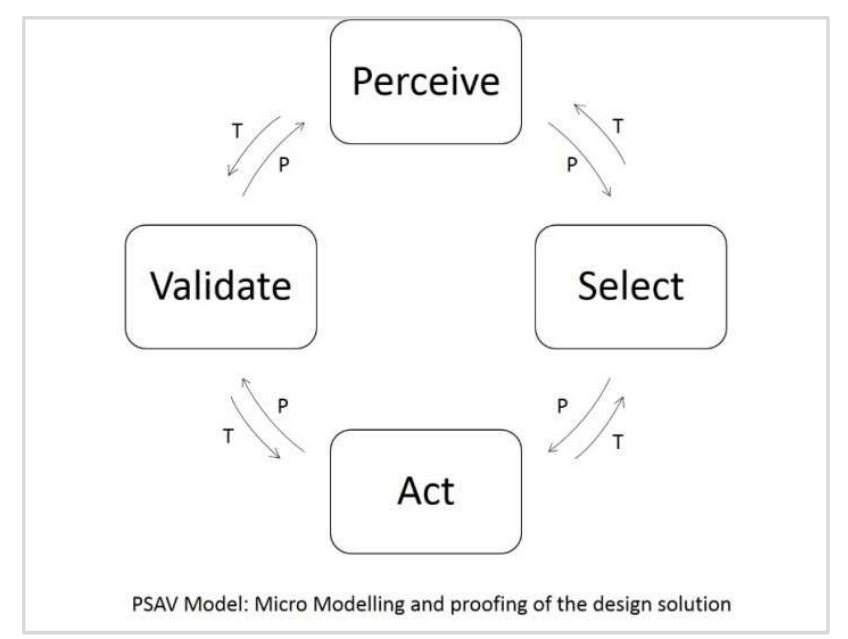

Figure 3:Micro Modelling and Proofing of the Design Solution

\section{CONCLUSIVE VALIDATION}

This section refers to the experiment published by the author for the application of Perceive -Select- Act- Validate method without actually describing the actual method. A set of process optimization exercises were conducted and recorded as a part of this experiment. The information collected from the experiment was further analyzed for its applicability. Process optimization is considered one of the pillars of the user experience design. PSAV model methodology was tested for improving the user experience in this set of experiment. As shown in the Figure 4 below, it can be observed that a significant reduction in the process steps were achieved through the PSAV model methodology application in user experience design process.

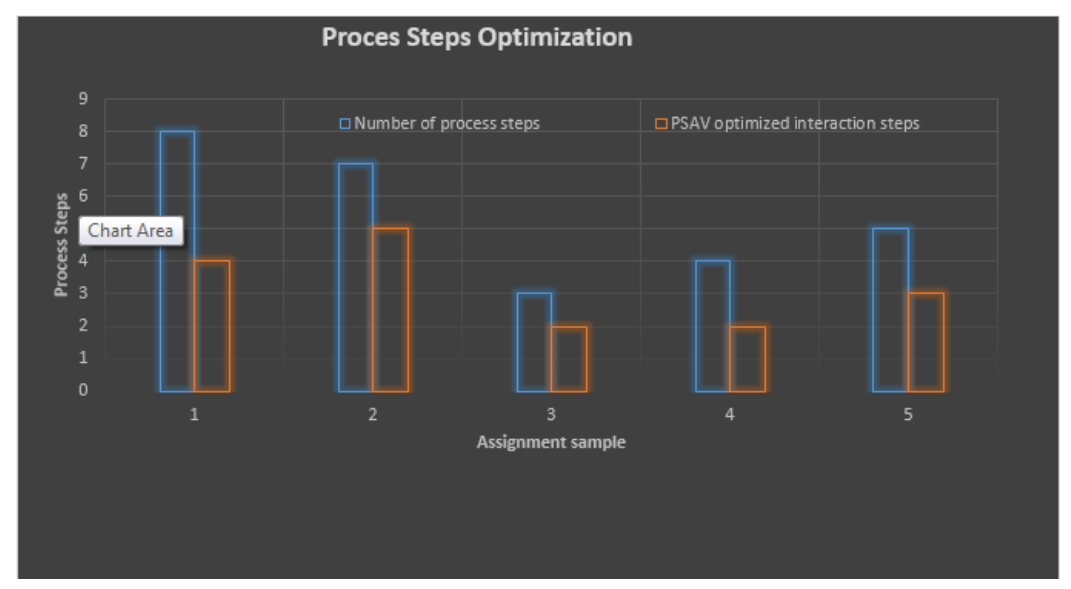

Figure 4: Result of Process Optimization Exercise

The output of the experiment supports the applicability of PSAV method in the area of user experience design. It shall be noted here that a well-defined problem statement and meaningful insights supports the agile implementation of the PSAV method. It was also empirical during the experiment that to apply the PSAV model contextual research or understanding is needed on the designer's part.

\section{APPLICATION AREAS}

Application of PSAV method can effectively be utilized in Human Computer Interaction Design, Interaction and interface design for IOT devices, Mobile web application, interface and interaction design, system design, medical device 
design, however not limited to. It is also helpful in the development phase of any project concerning the digital application of information technology.

\section{REFERENCES}

1. Elito Method, http://methods-journal.wikia.com/wiki/Elito

2. Universal Methods of Design: 100 Ways to Research Complex Problems, Develop Innovative Ideas, and Design Effective Solutions

3. Agile method,https://www.scruminc.com/scrum-blog/

4. KJ Technique, https://articles.uie.com/kj_technique/

5. Kano Aanalysis, https://en.wikipedia.org/wiki/Kano_model

6. The Gita-in contemporary times; Relevant and applicable for designers, 2018 JETIR May 2018, Volume 5, Issue 5 www.jetir.org (ISSN-2349-5162)

7. Determination of Inflation Steps for Rubber Inflatable Seal Using FE Analysis, International Journal of Engineering Science and Computing, December 201715694

8. Don't make me think, Steve Kurg, Pearson ISBN 978-93-325-4286

9. Application of PSAV method in interaction steps optimization for user experience design, IJARIIT ISSN: 2454-132X, Vol.4, Issue 5

\section{Author Details}

Author is the inventor of PSAV Method, he is an active design researcher and strategist. He is an alumnus of the Indian Institute of Technology Kanpur. He is currently associated with Tata Consultancy Services as a User Experience Analyst. Apart from futuristic design research, he is also an explorer of ancient scientific scriptures. He has published book chapters and research papers in journals of international repute. His research papers have helped several organizations to build strategic plans for product usability and user experience. 
